\title{
Two kinds of adaptation in the constancy of visual direction
}

\author{
HANS WALLACH \\ Swarthmore College, Swarthmore, Pennsvlvania 19081 \\ and \\ TERESA CANAL
}

Department of Human Development and Family Studies, Cornell University, Ithaca, New York 14853

\begin{abstract}
Adaptation in the constancy of visual direction had previously been obtained by causing a large or a small visible area representing the environment to be objectively displaced in dependence on head movements. No stationary objects were permitted to be visible. Now experiments are reported in which displacements of a large patterned field, with the subject fixating a stationary mark in its center, led to adaptation. In these experiments, objective displacements of the environment were given by image displacements on the retina. Adaptation also resulted when the large field was stationary and only the fixation mark was displaced. Here the objective displacement was given by the rate of pursuit eye movements.
\end{abstract}

When one turns one's head, the visual environment is being displaced relative to the eyes in the direction opposite to the head movement, but the stimulation that results from this displacement does not cause perceived motion of the environment. That this is due to a compensating process became clear when Stratton (1897) wore inverting lenses: Stratton saw his visual environment swing with every turning of the head in the direction of this movement. His lenses caused his visual environment to be optically displaced in the direction with the head movement, a condition for which there was no compensation. Stratton also found that, with time, he could adapt to this effect of his lenses and develop complete compensation for the displacement of the environment in the direction with the head movement. This adaptation did not only cause perception of a stationary environment when the lenses were worn, it also manifested itself in an apparent swinging of the visual field when he turned his head after removal of the lenses. This latter fact can also be used to demonstrate partial adaptation to optical field displacement during head turning that develops after short adaptation periods. Posin (1966) and Wallach and Kravitz (1965) employed this method to demonstrate small adaptation effects produced by exposing a subject to a visual target that was mechanically displaced by the subject's own head turning.

Wallach and Kravitz (1965) also developed a method for measuring the compensation accurately

This work was supported by Grant 11089 from the National Institutes of Health to Swarthmore College. Hans Wallach, principal investigator. and thereby opened the way for the quantitative investigation of adaptation to optical or mechanical displacement of the environment during head movements. Their method was based on a device that employed a variable ratio transmission to put the location of the scene in front of the subject under the control of his head position. The subject's head was attached to the input shaft of the transmission, and a mirror was mounted on its output shaft. That mirror reflected the beam of a projector on a screen in front of the subject where the projected scene was focused. When the subject turned his head, this arrangement caused the scene to shift sideways, at a rate that depended on the ratio to which the transmission was set. In other words, it was possible to vary the ratio between the angular displacement of the scene and the rate of rotation of the head. This ratio, called displacement ratio (DR) by Wallach and Kravitz, became the scale for measuring the accuracy of the compensation process and the amount of adaptation achieved.

The accuracy of the compensation process is measured by asking: How much can the projected scene in front of the subject move during a turning of the head either in the direction with the head movement or in the opposite direction, without that motion of the scene being perceived? Such a measurement involves setting the variable transmission for a displacement of the scene in the direction with the head movement and diminishing this displacement stepwise until the subject no longer sees the scene move. This is one limit of the subject's no-motion range. The procedure is repeated, starting with displacements of the scene in the direction against the 
head movement, and the other limit of the no-motion range is found. The interval between the two limits, the no-motion range, is a measure of the accuracy of the compensation process. (For a discussion of the methods used, see Wallach and Kravitz, 1968.)

To measure the amount of adaptation produced by optical or mechanical head-movement-dependent displacements of the environment, another nomotion range was measured after exposure to these adaptation conditions. The change in the location on the DR scale of the midpoints of the two no-motion ranges measured the adaptation effect. If the field displacement to which the subject adapts is, e.g., in the direction with the head movement, the field will afterwards appear stationary when it moves in some amount in the direction with the head, that amount being represented by the midpoint of the new nomotion range. Correlatively, a stationary bright spot in the dark will appear to move horizontally back and forth in the direction against the head movement when the subject turns his head. Because the method that measures the change of the no-motion range can be said to compensate for the apparent displacement of a stationary target, we shall call it compensation method.

Much effort has been made in investigating various aspects of this adaptation. For a list of previous work, see Wallach, Yablick, and Smith (1972). The experiments to be reported here are concerned with the nature of this adaptation. When one turns one's head under normal circumstances, it is usually to look at another part of the visual field. This involves saccadic eye movements in the direction of the head movement. But since saccades are faster than head movements, there are brief periods during which the eyes remain fixed on a spot in the visual field while it becomes displaced relative to the turning head. During these periods, slower eye movements take place that compensate for the head rotation. When, during the adaptation period, the visual field is being displaced dependent on the head movements, both kinds of eye movements have to change. If, for instance, the field displacement is in the same direction as the head rotation, the compensating eye movements have to be smaller than normal in relation to the head movements in order to stay fixed on the same point in the visual field, and the saccades that occur in conjunction with the head movements must also be smaller in order to land the gaze on the desired spot in the visual field. Adaptation to field displacement in direction with the head movements means that the nervous system accepts these diminished eye movements as denoting a stationary visual field, inasmuch as the moving field appears to be stationary when adaptation has taken place. ${ }^{1}$ It is then possible that the adaptation we are studying consists in an altered evaluation of the eye movements that mediate field displacements. If that were the case, it should not matter how the visual field moves during the adaptation period. Adaptation should occur if a fixation mark undergoes regular displacements during head turning. It is, on the other hand, possible that the condition that causes adaptation is the occurrence of regular field displacements during head movements and that adaptation consists in a changed evaluation of these field displacements, irrespective of how they are mediated. In that case, it might be possible to obtain adaptation when the visual field is regularly displaced during head movements while the eyes remain fixed on an objectively stationary mark, an arrangement that involves normal compensating eye movements taking place when the head is turned.

We tried, therefore, to obtain adaptation under two exposure conditions. In one, a large pattern was being displaced dependent on the head movements while the subject was under instruction to fixate a small stationary mark straight in front of him. We call an adaptation obtained under these conditions "field adaptation."' In the other exposure condition, the large patterned field was kept stationary and the fixation mark was made to displace dependent on the head movement. We call an adaptation obtained under the latter conditions "eye-movement adaptation."

\section{EQUIPMENT AND PROCEDURE}

The apparatus in which the subject adapted and was given the compensation test was briefly described above; a more detailed description can be found in Wallach and Kravitz (1968). For our experiments, it was modified to provide a large projected scene. The projector was equipped with a wide-angle lens. Along with it, the mirror on the output shaft of the transmission which shifted the scene back and forth dependent on the head movement was replaced by a larger one. The screen, which was $180 \mathrm{~cm}$ wide and $150 \mathrm{~cm}$ high, was $120 \mathrm{~cm}$ from the subject's eyes. There were three further changes: A second, stationary mirror was mounted at the base of the transmission and was used when there was need to project a stationary fixation mark or a stationary scene. Also, a flexible shaft was attached to the control shaft of the variable transmission. It ended in a knob that was mounted on the right armrest of the subject's chair. By turning this knob, the subject could alter the setting of the transmission himself and thereby adjust the displacement ratio (DR) with which the scene in front of him moved in dependence on the head movement. Finally, a different target spot was used, a dim circular spot of light of $5 \mathrm{~cm}$ diam and showing a grid of fine lines; the grid was produced by inserting a reticle into the slide with which the target spot was projected. The lines favored the spot to be viewed with accurate convergence and accommodation. The procedure of having the subject himself change the DR of the motion of the scene continuously was substituted for the abbreviated method of limits that had previously been used to measure adaptation by compensation. The new method still yielded the two limits of the no-motion range, and the change on the DR scale of the midpoint of the no-motion ranges obtained before and after the adaptation period was still the measure of the adaptation effect. 
The new method had the advantage of taking less time, and this diminished the chance that a decrease of the adaptation effect developed during the postadaptation test.

The displacement to which the subject adapted always amounted to $40 \%$ DR and was always in the direction with the head movement; Wallach, Frey, and Romney (1969) had obtained a stronger adaptation effect from that condition than from target displacement against the head-rotation direction. The adaptation period lasted always $10 \mathrm{~min}$.

The following procedure was used in all tests: In the preexposure test, the experimenter set the transmission so that the spot moved in the direction with the head movement by $4 \%$ DR. The subject was then asked to turn his head back and forth and report whether the spot appeared to move. All subjects saw the spot move in the direction with the head under this condition. Then the subject was asked to turn his head back and forth and slowly turn the control knob counterclockwise. This slowly lowered the transmission ratio and decreased the motion of the target spot. The subject was to stop turning the knob as soon as he saw the spot no longer move and then close his eyes. At that point, the experimenter read the setting of the variable transmission with a flashlight. She then changed the setting of the transmission so that the spot moved with 4\% DR in the direction against the head and asked the subject to observe the spot's motion while turning the control knob clockwise. Again, he was to stop when the spot no longer seemed to move, and the experimenter took another reading of the transmission setting. The two readings constituted the limits of the no-motion range.

Partial adaptation to the displacement of the scene means that an objective displacement in the direction with head rotation of some amount will be perceived as stationary. That means that after the exposure period the no-motion range could be expected to be shifted on the DR scale in the direction with the head movement. The initial setting in the postexposure test was $10 \%$ DR with the head movement. If, at this point, the subject reported seeing the target stationary or moving in the direction against the head movement, the initial setting was changed to $15 \%$ DR. As in the preexposure test, the subject lowered the transmission ratio until he no longer saw the spot move. After this setting was recorded, the experimenter further lowered the transmission ratio by $4 \% \mathrm{DR}^{2}$ This setting caused all subjects to see target motion in the direction against the head rotation. Now the subject gradually changed the transmission ratio to increase the objective target displacement in the direction with the head movement until the spot no longer seemed to move, and this setting was recorded. The two settings constituted the limits of the postexposure no-motion range.

This procedure involved always taking first the limit of the nomotion range toward experienced target motion in the direction with the head movement and the other limit, the one toward experienced target motion against the head rotation, in the second place. Since both the pre- and postexposure tests were conducted in this way, and since we were interested only in the difference of the locations of the pre-and postexposure no-motion ranges on the DR scale, this procedure was acceptable. It had the advantage that, in the postexposure test, it minimized the subjects' exposure to target displacements that caused perceived target motion in the direction against the head rotation. Such an exposure tends to diminish the effect of adaptation to displacements of the visual scene in the direction with the head movements. ${ }^{3}$

The procedure just described was used in all our experiments. They varied only with regard to features of the exposure conditions. These differentiating features will be described together with the presentation of the results. Three control experiments were performed in the course of our investigation; they will be reported first.

\section{CONTROL EXPERIMENTS}

(1) In our first experiment, we checked on the new testing procedure. As in many previous experiments, a small target spot was employed in the exposure period, the same that was used in the tests. Ten subjects participated. At the start of the exposure period, they were instructed to turn their heads back and forth and look at the target spot.

The mean of the midpoints of the no-motion ranges measured in the preexposure tests coincided exactly with objective target immobility, and the mean midpoint measured in the postexposure tests amounted to $6.8 \%$ DR in the direction with the head movement. The mean adaptation effect was therefore $6.8 \%$ DR and was significant at the .001 level.

(2) In our critical experiments, large patterned fields were employed in the exposure period. One of the patterns was $108 \mathrm{~cm}$ wide and $90 \mathrm{~cm}$ high. It will be called "the large field." The pattern consisted of darker and lighter vertical stripes of varying width. The large field subtended visual angles of 48.5 and $39.3 \mathrm{deg}$. In our second control experiment, it was substituted for the target spot in the exposure period and made to displace dependent on the head movements at the rate of $40 \% \mathrm{DR}$. The subjects were instructed to keep turning their heads and look at the pattern in front of them.

Fifteen subjects had a mean midpoint of $.4 \%$ DR in the direction against the head movement in the preexposure test and a mean postexposure midpoint of $6.5 \% \mathrm{DR}$ in the direction with the head movement. The adaptation effect of $6.9 \%$ DR was signigicant at the .001 level.

(3) The third control experiment was nearly identical with the second one. Only a smaller pattern was substituted for the large field. This "medium field" was $54 \mathrm{~cm}$ wide and $45 \mathrm{~cm}$ high. It was produced by putting an opaque frame over the slide that was used to project the large field.

Fourteen subjects gave a mean midpoint of $.3 \% \mathrm{DR}$ in the direction with the head movement prior to the exposure period and a mean midpoint of $11.2 \%$ DR in the same direction after exposure. The adaptation effect of $10.9 \%$ DR was significant at the .001 level. The difference between this value and the smaller adaptation effect obtained with the large field was significant $(p<.01)$. No attempt will be made to explain this last result.

\section{EXPERIMENT I}

This experiment demonstrated eye-movement adaptation. An objectively stationary environment 
Table 1

Mean Adaptation Effects in Percentage Displacement Ratio (\% DR) Measured Under Six Experimental Conditions

No. of

$\% \mathrm{DR}$ Subjects

Control Experiments

$1.5 \mathrm{~cm}$ spot
2. large ficid

3. medium field

Critical Experiments

1. Moving mark on stationary field

II. Stationary mark on moving field large medium

$\begin{array}{rr}6.8 & 10 \\ 6.9 & 15 \\ 10.9 & 14 \\ & \\ 2.6 & 20 \\ 4.0 & 15 \\ 2.9 & 15\end{array}$

was given by projecting "the large field" on the screen in front of the subject by way of the stationary mirror. Only a fixation mark, visible in the center of the large field, underwent the displacements dependent on the head movements. The target spot that had been used in the preexposure test remained visible to become the fixation mark and was given the displacement ratio of $\mathbf{4 0 \%}$. A second lantern, which projected the stationary field, was switched on for the duration of the exposure period. The subject was instructed to turn his head continuously and follow the small dot with his eyes. Twenty subjects participated.

Prior to exposure, the mean midpoint was .2\% DR in the direction with the head movement. After the exposure, that mean was $2.8 \%$ DR. The adaptation effect amounted to $2.6 \% \mathrm{DR}$ and was significant at the .001 level.

\section{EXPERIMENT II}

Two experiments were performed that demonstrated field adaptation. In one, the large field and, in the other, the medium field were employed. As in Control Experiments 2 and 3, the fields were made to displace dependent on the head movements, but eye movements were kept normal by giving the subject a stationary fixation mark to look at.

In this experiment, the beam of the wide-angle projector was reflected by a large mirror mounted on the output shaft of the variable transmission. During the tests, this lantern projected the target spot. For the exposure period, the slide for the target spot was replaced by the slide with the field pattern. Also, a second lantern was turned on, which projected, via the stationary mirror, a fixation spot of $1 \mathrm{~cm}$ diam and equipped with a grid. Fifteen subjects adapted to displacements of the large field.

The mean midpoint changed from .1\% DR before to $4.1 \%$ DR after the exposure period. The adaptation effect of $4 \%$ DR was significant at the .001 level.
When, in this experiment, the large field was replaced by the medium field, another group of 15 subjects, whose mean preexposure midpoint had also amounted to $.1 \% \mathrm{DR}$, gave an adaptation effect of $2.9 \%$ DR, significant at the .001 level.

\section{DISCUSSION}

We have shown that adaptation in the constance of visual direction can be obtained under two novel conditions that are radically different from each other. The ordinary adaptation conditions, whose effects had been investigated up to the present, were represented in our work by the three control experiments. A small or a large part of the visual environment was visible and was being objectively displaced during and dependent on head movements, but no stationary objects or spots could be seen. When only a small target spot was displaced, the remainder of the visual field was totally dark. In our two critical experiments, only part of the visible environment underwent the displacement; some visible part was stationary. In the condition that, we felt, leads to eyemovement adaptation, only a small target spot was being displaced and a large part of the environment was visible and stationary. In the field-adaptation condition, where a large part of the environment was seen being displaced, a small stationary spot was visible and served as fixation mark. Eye movements were, therefore, normal in this condition and could not have been the vehicle for the field displacement. While highly significant adaptation effects were obtained under these critical conditions, the magnitude of the effects was small.

Table 1 shows the adaptation effects obtained in our six experiments. The results of Control Experiments 1 and 2 are in good agreement with each other, with the adaptation effect amounting to about $6.8 \%$ DR. When the adaptation effects obtained after field adaptation are added to the effect of eye-movement adaptation, the result has approximately the same value. It is tempting to assume that the effect of the ordinary adaptation conditions is a summation of two adaptation processes, one in which the objective displacements in the visual environment are given through image displacements on the retina (field adaptation) and the other in which it is given through pursuit eye movements of points in the displacing environment at which the subject looks while turning his head. Whether this interpretation of ordinary adaptation in the constancy of visual direction is accepted depends on one's view of Control Experiment 1. Here the visible part of the environment consisted of a spot with a diameter of only $5 \mathrm{~cm}$, subtending a visual angle of $2.4 \mathrm{deg}$. The question is whether such a small object is viewed with perfect pursuit movements or whether image displacements that can produce field adapta- 
tion also occur. If only perfect pursuit movements are assumed to occur, the question arises as to why the adaptation effect in Control Experiment 1 was significantly larger $(p<.01)$ than the one in Critical Experiment I. In that case, the presence of a large stationary field in the conditions of Experiment 1 must be assumed to have a retarding effect on the adaptation due to eye movements. In one way or another, our two adaptation processes, field adaptation and eye-movement adaptation, seem to interact. Either their effects summate or contradictory conditions for field adaptation diminish the development of eye-movement adaptation.

\section{REFERENCES}

Posin. R. Percepraal adaptation to contingent visual-field movement: $A_{n}$ experimental investigation of position constancy. Doctoral Dissertation. Yeshiva University, 1966.

Stratton. G. M. Vision without inversion of the retinal image. Psychological Revi'w. 1897, 4. 341-360, 463-481.
Wallach, H., Frey. K. J.. \& Rommey. G. Adaptation to ficld displacement during head movement unrelated to the constancy of visual direstion. Perception \& Psychophysics. 1969. 5. 253-256.

Wallach. H.. \& Kravitz, J. The measurement of the constancy of visual direction and of its adaptation. Psychonomic Science, 1965. 2, 217.218.

Wallach, H., \& Kravitz, I. Adaptation in the constancy of visual direction tested by measuring the constancy of auditory direction. Perception \& Psychophysics, 1968, 4, 299-303.

Wallach, H., Yablick, G. S., \& Smith, A. Target distance and adaptation in distance perception in the constancy of visual direction. Perception \& Psychophysics, 1972. 12. $139 \cdot 145$.

\section{NOTES}

1. For simplicity's sake, we refer here to complete adaptation, although our experiments were concerned with partial adaptation.

2. This new initial setting could be possibly one in which the target moved objectively in the direction against the head rotation.

3. See, for instance, Experiment 1 in Wallach, Frey, and Romney (1969).

(Received for publication November 7, 1975: accepted February 9, 1976.) 